\title{
Helmholtz Stereopsis on rough and strongly textured surfaces
}

\author{
Jean-Yves Guillemaut ${ }^{1}$, Ondřej Drbohlav ${ }^{2}$, Radim Šára $^{3}$ and John Illingworth ${ }^{1}$ \\ ${ }^{1}$ Centre for Vision, Speech, and Signal Processing, University of Surrey, UK \\ ${ }^{2}$ Texture lab, School of Mathematical and Computer Sciences, Heriot-Watt University, UK \\ ${ }^{3}$ Center for Machine Perception, Czech Technical University in Prague, CZ
}

\begin{abstract}
Helmholtz Stereopsis (HS) has recently been explored as a promising technique for capturing shape of objects with unknown reflectance. So far, it has been widely applied to objects of smooth geometry and piecewise uniform Bidirectional Reflectance Distribution Function (BRDF). Moreover, for non-convex surfaces the inter-reflection effects have been completely neglected. We extend the method to surfaces which exhibit strong texture, nontrivial geometry and are possibly non-convex. The problem associated with these surface features is that Helmholtz reciprocity is apparently violated when point-based measurements are used independently to establish the matching constraint as in the standard HS implementation. We argue that the problem is avoided by computing radiance measurements on image regions corresponding exactly to projections of the same surface point neighbourhood with appropriate scale. The experimental results demonstrate the success of the novel method proposed on real objects.
\end{abstract}

\section{Introduction}

Helmholtz Stereopsis (HS) has recently been explored as a method which is able to reconstruct 3D shape of objects with unknown reflectance. The method exploits reciprocity of reflectance which states that the Bidirectional Reflectance Distribution Function (BRDF) [4] stays invariant when the incident and reflected directions are interchanged. The method has been applied successfully both in multicamera setups $[3,8,9]$ as well as in the binocular configuration $[10,7]$.

For all implementations presented so far, it is a matter of fact that the surfaces reconstructed have been smooth and of piecewise uniform BRDF. In reality however, many objects deviate from having such properties. In this article we address the case when the surface of an object reconstructed is rough or strongly textured (i.e. of fast spatial variation of BRDF). In addition, we observe that HS is affected by inter-reflections in case that the object is non-convex.

We argue that the standard version of HS which con- structs radiometric constraints based on single pixel measurements in images can fail on such objects. After further insight into the physics of reflection, we propose a novel method which is able to produce correct unbiased results. The main idea is to compute the radiance measurements over more extended regions instead of single pixels. Using extended regions corresponding to the projection of the same surface point neighbourhood maintains the validity of Helmholtz reciprocity at a macroscopic level at the expense of loosing the microscopic geometrical resolution.

\subsection{Overview of Helmholtz Stereopsis}

Before illustrating the problem, let us begin with a review of the HS principle. Consider the following setup. Let $\mathbf{O}_{l}$ and $\mathbf{O}_{r}$ be two points in space and $\mathbf{X}$ be a point on a surface. Let the surface orientation at $\mathbf{X}$ be $\mathbf{n}$ (the notation is the same as in Fig. 1 which will be explained later). The BRDF $f(\mathbf{u}, \mathbf{v})$ of a surface point is by definition the ratio of the outgoing radiance along the direction $\mathbf{v}$ to the incident irradiance along the direction $\mathbf{u}$. Let an isotropic light source of intensity $\kappa$ and a camera be positioned respectively at $\mathbf{O}_{l}$ and $\mathbf{O}_{r}$, and let $d_{l}=\left\|\mathbf{O}_{l}-\mathbf{X}\right\|$ and $d_{r}=\left\|\mathbf{O}_{r}-\mathbf{X}\right\|$ be their respective distance from the surface point $\mathbf{X}$. The pixel intensity ${ }^{1} i_{r}$ observed by the camera is

$$
i_{r}=f\left(\mathbf{i}_{l}, \mathbf{i}_{r}\right) \frac{\mathbf{i}_{l} \cdot \mathbf{n}}{d_{l}^{2}} \kappa
$$

where $\mathbf{i}_{l}=\left(\mathbf{O}_{l}-\mathbf{X}\right) / d_{l}$ and $\mathbf{i}_{r}=\left(\mathbf{O}_{r}-\mathbf{X}\right) / d_{r}$ are unit vectors along respectively the incident light ray and the outgoing ray at $\mathbf{X}$ (see Fig. 1). If the positions of the light source and the camera are now interchanged ${ }^{2}$, an analogous formula can be stated about the radiance $i_{l}$ observed by the camera at position $\mathbf{O}_{l}$ :

$$
i_{l}=f\left(\mathbf{i}_{r}, \mathbf{i}_{l}\right) \frac{\mathbf{i}_{r} \cdot \mathbf{n}}{d_{r}^{2}} \kappa
$$

\footnotetext{
${ }^{1}$ We adopt the convention that pixel intensity equals the scene radiance. We performed radiometric calibration of a camera in order to meet this requirement.

${ }^{2}$ Note that the same light source with the same intensity $\kappa$ is used.
} 
The two configurations are reciprocal; we say that the two images observed by such cameras form a reciprocal pair. Helmholtz reciprocity requires that $f\left(\mathbf{i}_{l}, \mathbf{i}_{r}\right)=f\left(\mathbf{i}_{r}, \mathbf{i}_{l}\right)$. Denoting $\mathbf{s}_{l}=\mathbf{i}_{l} / d_{l}^{2}$ (and analogously for $\mathbf{s}_{r}$ ), Equations (1) and (2) can be combined to obtain [8]

$$
\left(i_{l} \mathbf{s}_{l}-i_{r} \mathbf{s}_{r}\right) \cdot \mathbf{n}=0 \text {. }
$$

This equation represents the Helmholtz radiometric constraint which has been used as a matching constraint in previous work [3, 8, 9, 10,7]. The outstanding feature of this constraint is that it uses only a non-parametric property of the BRDF (the Helmholtz symmetry) but does not make any use of the actual BRDF values.

If $n$ constraints defined in Eq. (3) (one for each reciprocal pair) are stacked into one matrix, we obtain

$$
W \mathbf{n}=\mathbf{0} \quad \text { with } \quad W=\left[\begin{array}{c}
\left(i_{l_{1}} \mathbf{s}_{l_{1}}-i_{r_{1}} \mathbf{s}_{r_{1}}\right)^{\top} \\
\left(i_{l_{2}} \mathbf{s}_{l_{2}}-i_{r_{2}} \mathbf{s}_{r_{2}}\right)^{\top} \\
\ldots \\
\left(i_{l_{n}} \mathbf{s}_{l_{n}}-i_{r_{n}} \mathbf{s}_{r_{n}}\right)^{\top}
\end{array}\right] .
$$

If the intensities used for constructing the matrix $W$ come from a point which is located at an object surface, the matrix is ideally of rank 2. Hence a suitable measure of rank of $W$ can be used to discriminate between surface and nonsurface points [8]. We call saliency the entity employed and define it in terms of the second and third singular values $\sigma_{2}$ and $\sigma_{3}$ of $\boldsymbol{W}$ (it is assumed here that the singular values are positive and in descending order):

$$
r=\frac{\sigma_{2}-\sigma_{3}}{\sigma_{2}}
$$

The normal $\mathbf{n}$ at a point is evaluated as the third singular vector. A similar measure has been used for its simplicity in previous work $[3,8,9]$.

In the next sections, we show that this radiometric constraint is biased when the surface is rough or highly textured, and propose a unified approach to these problems.

\section{Rough and Textured Surfaces}

In this section we illustrate on elementary examples that the Helmholtz radiometric constraint from Eq. (3) is affected by inter-reflections and the presence of strong texture when single pixel measurements are used to construct it.

Rough Surfaces. Rough surfaces are microscopically non-convex and accommodate multiple reflections. Let us consider an example of a non-convex scene accommodating inter-reflections (see Fig. 1). The scene consists of just two planar patches, one of which (denoted by $\mathbf{M}$ ) is a perfect mirror. While the intensity observed by a camera at position $\mathbf{O}_{r}$ in the incident direction $\mathbf{i}_{r}$ (cf. Fig. 1(left)) is not altered by the presence of the mirror-reflecting patch, the intensity observed at the reciprocal configuration (cf. Fig. 1(right)) $i s$. As the intensity $i_{r}$ stayed fixed whereas $i_{l}$ was altered when introducing the mirror surface, this implies that the constraint from Eq. (3) does not correctly constrain anymore the normal direction $\mathbf{n}$ at point $\mathbf{X}$.
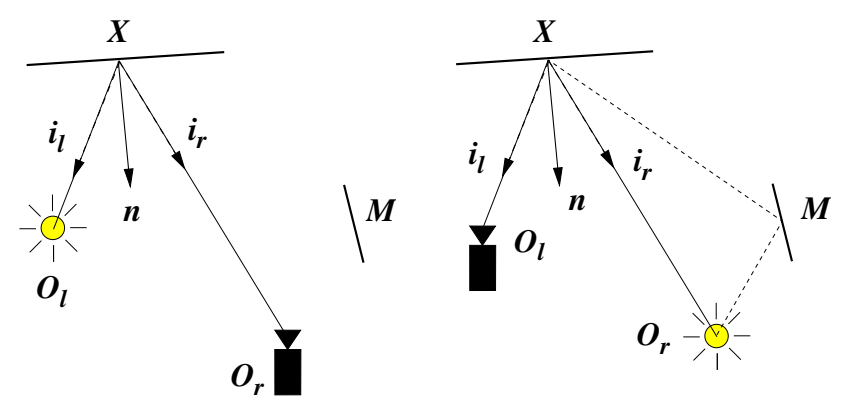

Figure 1: The illustration of the inter-reflection effect with concave scenes. The patch denoted $\mathbf{M}$ is a perfect mirror.

Highly Textured Surfaces. Similarly, if an object is strongly textured then single-pixel intensity measurements apparently violate reciprocity. The principal difficulty emerges from that the portion of a surface which an individual pixel observes varies as the camera changes its position and orientation in space. This problem is demonstrated in Fig. 2. Suppose for simplicity that the surface observed is Lambertian [2] and the variable surface albedo $\rho$ is either 0 (shown in black) or 1 (shown in white). A camera in a position according to Fig. 2(left) perceives a patch of albedo $\rho=1$ while a camera in configuration according to Fig. 2(right) would see $\rho \approx 1 / 3$.

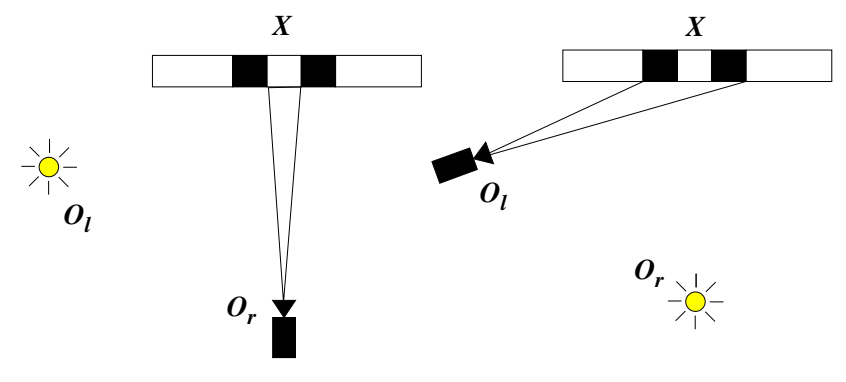

Figure 2: Observing different portions of the surface causes apparent violation of surface reciprocity.

\subsection{A Unified Solution}

For smooth and textured surfaces discussed at the end of the last section, the solution we propose is straightforward. One 
immediately sees that what is needed is to guarantee a consistent measurement, i.e. whatever the position of the camera is, to measure the radiance of the same physical surface patch. Technically, this involves averaging image regions corresponding to projection of an extended neighbourhood of $\mathbf{X}$ :

$$
I_{l}=\frac{1}{\mathcal{P}_{l}} \int_{\mathcal{P}_{l}} i_{l}, \quad I_{r}=\frac{1}{\mathcal{P}_{r}} \int_{\mathcal{P}_{r}} i_{r},
$$

where $\mathcal{P}_{l}$ and $\mathcal{P}_{r}$ are the areas of projection of the point neighbourhood into the left and right images respectively. In the case of textured surfaces, the reciprocity clearly holds at any point of the neighbourhood, and therefore its validity is preserved when such an averaging is done, such that an equivalent of Eq. (3) can be formulated for the integrated intensities $I_{l}$ and $I_{r}$ :

$$
\left(I_{l} \mathbf{s}_{l}-I_{r} \mathbf{s}_{r}\right) \cdot \mathbf{n}=0 .
$$

It is now shown that the same result also applies to rough surfaces. So far, when defining the BRDF, it has usually been assumed that the surface is smooth and homogeneous. It has only been proved recently in [5] that the principle holds in the case of BRDF corresponding to surfaces exhibiting non-trivial structure. The idea is that when the surface is rough then its macro-shape is represented by a reference plane (see Fig. 3). It can be easily shown, at least within scope of geometric optics (see [5]), that if the surface exhibits reciprocal behaviour at a microscopic level then any optical path passing through the structure is reciprocal. As a result, the BRDF of a surface patch defined as a ratio of average radiance to the reference plane irradiance is reciprocal, up to boundary effects caused by optical paths for which the incident ray enters the surface outside the patch and leaves inside it (or similarly, when the incident ray enters inside the point neighbourhood and leaves outside).

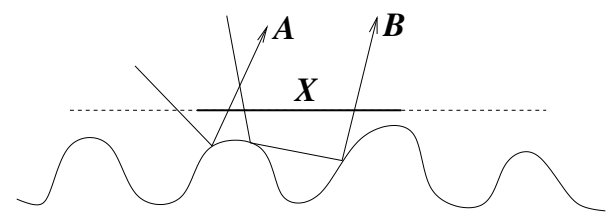

Figure 3: The concept of a reference plane (dashed) of a rough surface. Two optical paths are shown, one of which (A) contributes to boundary effects as it enters outside the extended neighbourhood of $\mathbf{X}$ (shown as a thick line segment) and leaves inside it.

This means that the radiance measurements constructed according to Eq. (6) preserve reciprocity provided that the boundary effects are negligible. In practice, the impact of boundary effects can be decreased by averaging over more extended surface point neighbourhoods. This result is applicable to both textured and rough surfaces.

\subsection{Validation of the Principle}

To demonstrate the validity of this result, we conducted an experiment with a concave object exhibiting strong interreflections. The object used was a hemispherical concavity realised by sectioning a white ping-pong ball. A reference plane was defined by the great circle at a place of the cut, a point of interest $\mathbf{X}$ was the sphere centre and the extended neighbourhood of $\mathbf{X}$ was defined by the outline of the cut (see Fig. 4). For such configuration, no boundary effects existed indeed, as no rays were allowed to enter outside the extended neighbourhood and leave inside, or vice versa. In an experiment, Helmholtz image pairs of the ping-pong ball section (see Fig. 5) were acquired. The experimental set-up used is described in Section 4. Five different sets made of eight reciprocal pairs each were obtained for five different inclination angles of the ping-pong ball section (cf. Fig. 4).
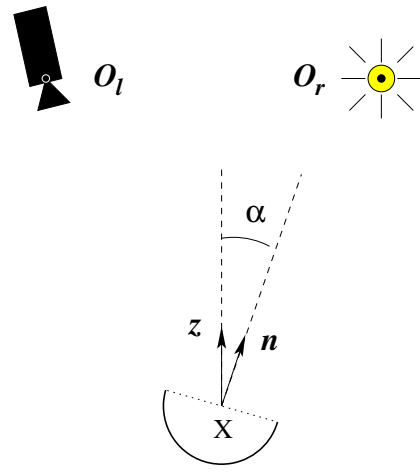

Figure 4: Experimental setup for the ping-pong ball section. The normal $\mathbf{n}$ of the ball is inclined by an angle $\alpha$ with respect to the vertical direction $\mathbf{z}$. The reference plane at $\mathbf{X}$ corresponds to the plane of the cut.

We show that with the novel method introduced earlier, it is possible to obtain accurate information about the macrostructure, i.e. here about the orientation of the ball section, even if a complete reconstruction is not possible. For each set of images we integrated the radiance within the region defined by the outline of the ping-pong ball in each image and normalised with respect to the extent of each region. This provided us with the integrated radiance measurements $I_{l}$ and $I_{r}$. Using the prior information concerning the position of the ball, the normal of a reference plane was computed using Eq. (7). The measured intensities allowed us to compute the vectors $I_{l} \mathbf{s}_{l}-I_{r} \mathbf{s}_{r}$ which constrained the normal direction $\mathbf{n}$. The normal $\mathbf{n}$ was then computed from the set of such vectors using Singular Value Decomposition (SVD) as in [8].

Consequently, the recovered normal was compared to a ground truth normal obtained by performing conventional stereo on the outlines of the cut of the ball. The experiments were carried out for five different orientations of the hemi- 

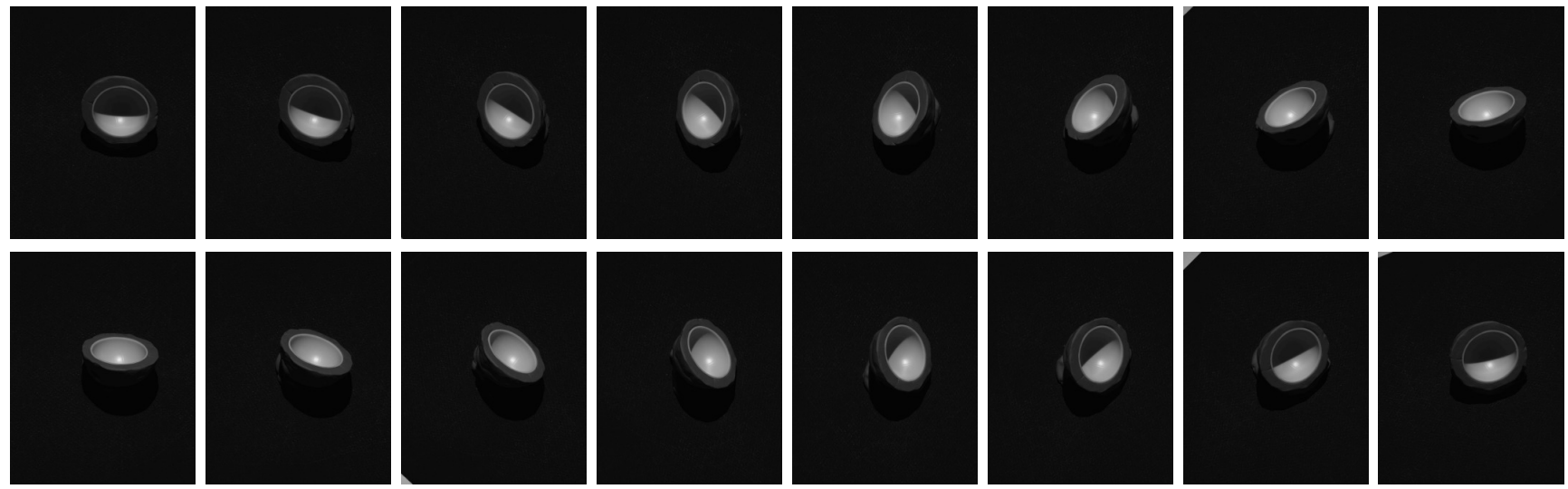

Figure 5: One set of eight reciprocal pairs of images of a ping-pong ball section (inclination angle $\alpha \approx 45$ ). The bottom row images are obtained by interchanging the position of the light source and camera with respect to the top row image. The outer shell of grey values is the clay that is holding the half ping-pong ball and also ensures that there are no transparency effect perturbing the experiment.

spherical concavity which differed in the inclination angle $\alpha$ of the ball section.

The comparison of the ground truth and the normal direction obtained from radiometric measurement is shown in Table 1. The results exhibit a good agreement. To evaluate the consistency of the radiometric constraint from Eq. (7) provided by the set of Helmholtz pairs, we computed the angular distance of the constraining vectors $I_{l} \mathbf{s}_{l}-I_{r} \mathbf{s}_{r}$ from the plane perpendicular to the recovered normal $\mathbf{n}$. The set of constraints is again consistent, as shown in Table 2. As the theory predicted, if radiance measurements are computed on image regions corresponding to the projection of bounded surface patches with appropriate scale then the radiometric constraint from Eq. (7) can be used to construct an accurate estimate of the patch geometry.

\begin{tabular}{cccccc}
\hline \hline Exp & 1 & 2 & 3 & 4 & 5 \\
\hline$\alpha_{G T}$ & 2.9 & 17.7 & 36.1 & 45.0 & 54.5 \\
\hline$\alpha_{\text {Int }}$ & 3.7 & 15.1 & 37.3 & 46.7 & 56.7 \\
\hline$\theta$ & 3.8 & 2.8 & 1.3 & 2.7 & 2.6 \\
\hline
\end{tabular}

Table 1: Comparison of the inclination angles obtained by the novel method using consistent measurements of radiance $\left(\alpha_{\text {Int }}\right)$ with the ground truth values $\left(\alpha_{G T}\right) . \theta$ is the angular difference between the ground truth normals and the normals computed by the method proposed. All values are in degrees.

\section{Implementation}

As it follows from the previous Section, the implementation of HS for rough and highly textured surfaces requires

\begin{tabular}{cccccc}
\hline \hline Exp & 1 & 2 & 3 & 4 & 5 \\
\hline RMS & 0.25 & 0.22 & 0.15 & 0.16 & 0.15 \\
\hline $\max$ & 0.39 & 0.35 & 0.27 & 0.36 & 0.22 \\
\hline \hline
\end{tabular}

Table 2: Root Mean Squared (RMS) and maximum deviation angle of the vectors $\left(I_{l} \mathbf{s}_{l}-I_{r} \mathbf{s}_{r}\right)$ from the plane orthogonal to the normal (eight vectors were used to compute the deviation). All values are in degrees.

to construct consistent measurements of surface radiance. Optimally, these measurements are computed by averaging intensity values over image regions corresponding to the projection of the same physical surface patch, as described by Eq. (6). Such construction, however, is non-trivial as for the evaluation of projections it would require the reference plane orientation which is not known in advance. We propose two different algorithms.

The first algorithm is called integrated $H S$ and uses an approximation of the exact radiance evaluation. The approximation is based on simple isotropic filtering of the image by a selected convolution kernel. Although Eq. (6) proposes simple averaging, we use a Gaussian kernel instead in order to down-weight the distant neighbourhood of the point in question. The choice of the kernel parameters is related to the scale of the surface structure or texture subelements. Currently these parameters are set empirically. Such an algorithm using isotropic filtering of the images is simple and runs very fast as the filtering can be done as a pre-processing step.

The second algorithm we propose is called adaptive $H S$. The main idea is to dynamically improve the averaging in 
Eq. (6). The integrated HS algorithm provides an estimate of the reference plane orientation which is used to initialise the adaptive HS algorithm. This normal estimate is used to compute the projection of a disc centred at the depth provided by the integrated algorithm. A refined normal is then computed rewriting Eq. (7) with the intensities integrated over the projection of the disc. We iterate until the change in the orientation of the normal estimated is inferior to a certain threshold $\left(0.1^{\circ}\right.$ in our implementation) or the maximum number of iterations is exceeded (10 in our implementation). As for the previous algorithm, the choice of the radius of the disc projected is dictated by the scale of the surface structure or texture sub-elements; this parameter is currently set empirically. In terms of run-time, the adaptive HS algorithm is slower than the integrated HS algorithm because it is iterative and also because the computation of the projection of a disc is more computer intensive. The adaptive HS algorithm is however expected to give more accurate results because it averages the intensities over areas corresponding to the projection of the same surface point neighbourhood.

\section{Experimental Results}

In this section we demonstrate the method generalised to textured and rough surfaces on two real objects. Obtaining the data and evaluating the 3D object geometry from radiance measurements is done similarly as in [8], with the only exception that we use the consistent measurements of radiance. The experimental setup consists of a camera, a light source and a turn-table which performs the interchange of camera and light positions. A 12 bit digital camera Vosskuhler CCD-1300 equipped with a $25 \mathrm{~mm}$ lens was used along with the halogen lamp acting as a point light source. Both the camera and the light source were radiometrically calibrated using the method described in [1]. The distance between the camera and the centre of the table is approximately $80 \mathrm{~cm}$ and the distance between the camera and the light source $60 \mathrm{~cm}$. Eight reciprocal pairs were used in the experiments to construct the radiometric constraints enabling to recover the $3 \mathrm{D}$ shape.

\subsection{Rough Surfaces: Teddy Bear}

We consider the reconstruction of a teddy bear (see Fig. 6). This is an example of very challenging object where traditional reconstruction algorithms (including standard HS) normally fail because of the anisotropic nature of the surface and the inter-reflection effects occurring between the hair of the fur. Fig. 7 shows the results of the reconstruction with and without integration. A Gaussian convolution kernel of size $21 \times 21$ pixels with standard deviation 4 pixels was used for the integrated HS algorithm. The radius of the patches used for projection was set to $3.5 \mathrm{~mm}$ for the adaptive HS algorithm. The choice of the size is dictated by the roughness of the surface of the teddy bear. A visual observation showed that such sizes lead to an appropriate smoothing of the images. In the case where integration is performed, the results obtained with the integrated HS and the adaptive HS are very similar; no qualitative difference was immediately visible, so we chose to only show the images of the reconstruction with the integrated HS algorithm (quantitative results will however be given for both algorithms later on in Table 3). The observation of the depth map, normal field and saliency map show a significant improvement when integration is performed. We observe that the integrated HS algorithm results in a less noisy depth map and normal field than for the standard HS algorithm. Larger saliency values can also be observed with the integrated HS algorithm when comparing the saliency maps.
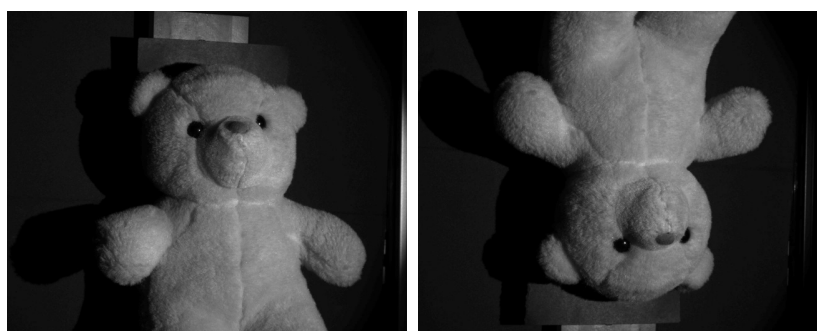

Figure 6: A reciprocal pair of images of the teddy bear; the second image is obtained by interchanging the position of the light source and camera.

The background contains a large number of inaccurately reconstructed points (see Fig. 7), so it was necessary to segment it from the object. The main motivation is to eliminate the unreconstructed regions which are due to discontinuities between the object and the background. It has been mentioned in [8] that these regions could be identified based on the correspondence between shadowed and half-occluded regions, however no implementation has been presented so far. In our approach we use the saliency map; background points adjacent to the object in the images have a lower saliency than object points (see Fig. 7). The saliency map is therefore a convenient feature for segmentation. Currently the segmentation algorithm is interactive.

Quantitatively, we define a measure of the quality of the reconstruction based on the saliency map called Root Mean Squared (RMS) saliency. The value is computed only over the points which belong to the object (i.e. after segmentation). Denoting by $N$ the number of such points, the RMS saliency is defined by: RMS $=\sqrt{\frac{1}{N} \sum_{i} \sum_{j} r_{i j}^{2}}$. The values obtained with and without integration are presented in Table 3; the quantitative results confirm that the saliency is increased with the two methods performing integration 

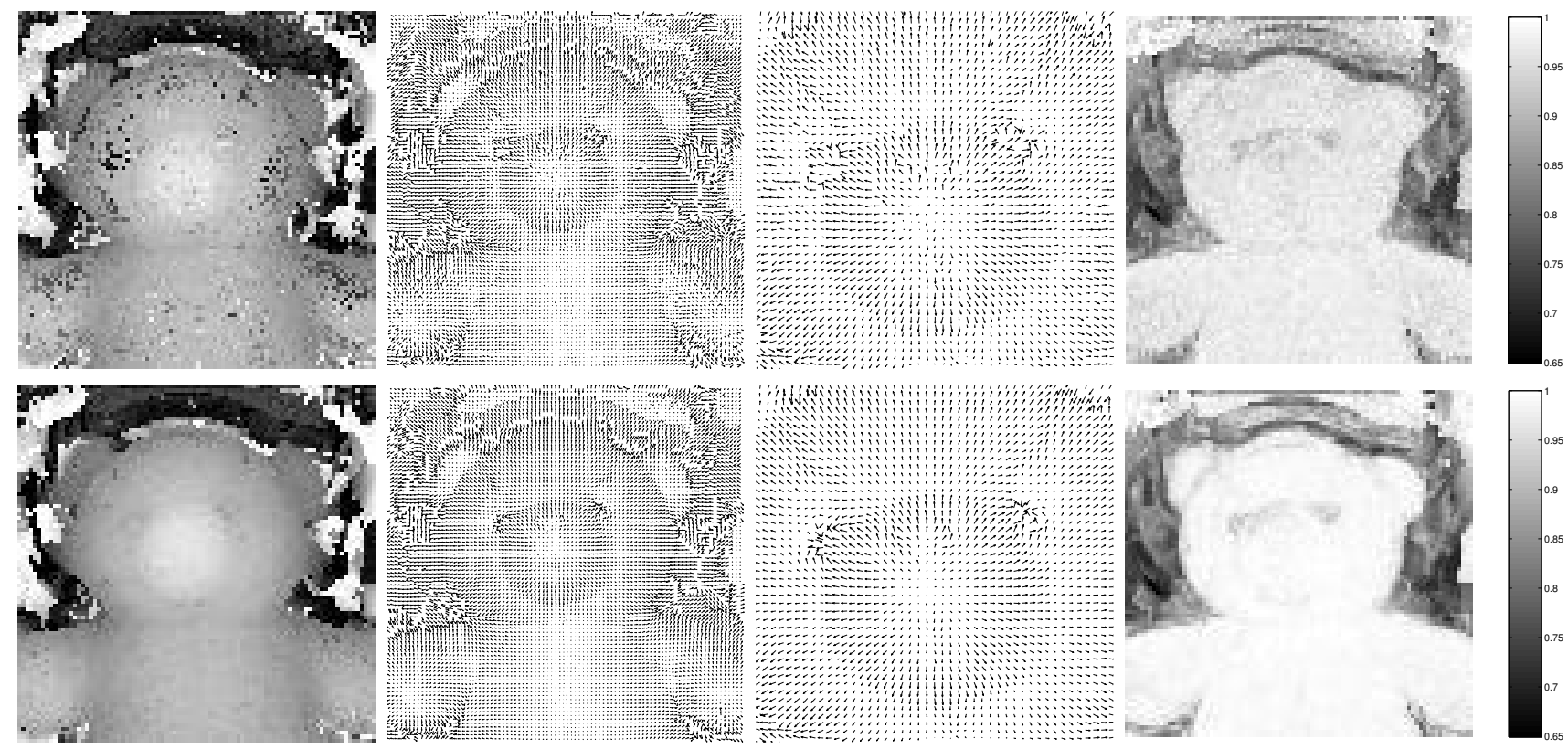

Figure 7: Reconstruction of the teddy bear using the standard (top row) and integrated (bottom row) HS algorithms: depth map (first column), normal field (second column), detail of the normal field for the face (third column), and saliency map (fourth column). The depth map and normals are less noisy when consistent measurements of radiance are used. Note also the increase in saliency.

(integrated and adaptive HS algorithms) compared to the standard HS algorithm. The integrated and adaptive HS algorithms lead to very close results in this case. Such an increase is important because it means that the novel method is able to produce more consistent models, which suggest that the method is more appropriate than the standard method. The integrated HS and adaptive HS algorithms give very close results.

\begin{tabular}{ccc}
\hline \hline & teddy bear & textured mug \\
\hline standard HS & 0.962 & 0.962 \\
\hline integrated HS & 0.985 & 0.989 \\
\hline adaptive HS & 0.984 & 0.988 \\
\hline \hline
\end{tabular}

Table 3: Comparison of the Root Mean Squared (RMS) saliency obtained with the reconstruction of the teddy bear and the textured mug in the case of the standard HS, the integrated HS and the adaptive HS algorithms. A high saliency suggests that the method is able to produce consistent models and that the reconstruction is therefore appropriate.

Typically the normal field can be estimated more accurately than the depth map [8], for this reason a 3D model of the surface is obtained from integration of the normal field using an approach similar to [6]. The method has the advantage of using weight coefficients during integration, which allows us to incorporate elegantly the background segmentation results and also weight the surface points by their saliency. The 3D model obtained with the standard and the integrated HS algorithm are shown in Fig. 8. The integrated HS algorithm is able to reconstruct accurately the shape of the object at a marcrosopic level, including some small details such as for example the seam on the belly or the nose. The model appears to be smoother than with standard HS algorithm. The model can also be texture mapped with one input image to produce a realistic rendering of the teddy bear.

\subsection{Textured Surfaces: Mug}

The object chosen to demonstrate the method in the case of strongly textured surfaces is a mug. The object accommodates a texture made of blue dot patterns (see Fig. 9). The results obtained with the standard and integrated HS algorithms are shown in Fig. 10. A Gaussian kernel of size $21 \times$ 21 pixels with standard deviation 4 pixels was used for averaging in the integrated HS algorithm. We observe that the integrated HS algorithm results in a less noisy depth map and normal field, and an improvement in the saliency of the reconstruction. A normal field integration scheme using object segmentation and weighting by the saliency as de- 


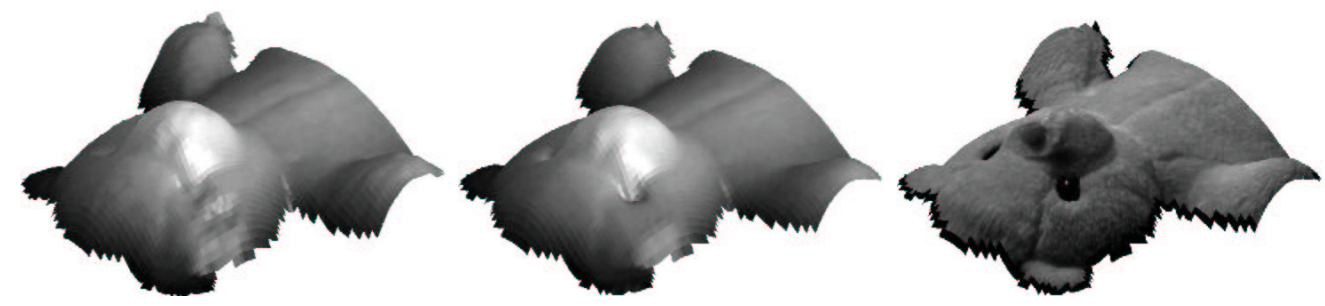

Figure 8: The surface of the teddy bear reconstructed with the standard HS algorithm (left) and integrated HS algorithm (centre). The surfaces are obtained by integrating the normal fields shown in Fig. 7. The image on the right shows the surface reconstructed with the integrated HS algorithm and texture mapped with the left image from Fig. 6.

scribed in the previous paragraph leads to accurate and realistic geometric models (Fig. 11). The reconstruction was also carried out with the adaptive HS algorithm (with a radius of $4 \mathrm{~mm}$ for the projected discs), and results very similar to the integrated HS method were obtained. The quantitative results based on computation of the RMS saliency confirm that the integrated HS and the adaptive HS algorithms produce more consistent results than the standard HS algorithm. Again it can also be noticed that the results of the two algorithms performing integration are very close.
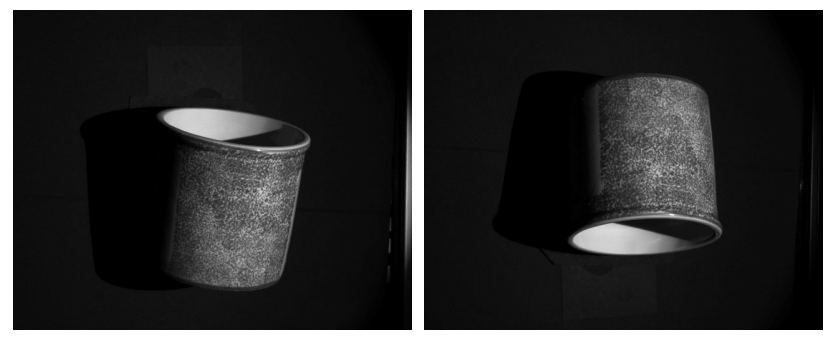

Figure 9: A reciprocal image pair of a textured mug.

\section{Summary and Conclusions}

Rough and highly textured surfaces are often encountered in reality. The possibility to reconstruct their shape is important in computer vision. In this work we explicitly addressed the problem of reconstructing such surfaces by Helmholtz Stereopsis (HS). We observed that radiometric constraints constructed from single pixel measurements are necessarily biased when inter-reflections or strong texture are present. We showed that a solution is to construct consistent measurements from image regions corresponding to the projections of the same bounded surface patch instead. A thorough experiment on a hemispherical concavity revealed good agreement of the results with the theory.

Two different HS algorithms generalised to highly textured and rough surfaces were proposed. The first algo- rithm, called integrated $H S$, approximates the integration by pre-processing each input image using isotropic filtering. This is equivalent to running the standard HS algorithm on the pre-convoluted input images. As such, consistent measurements can be obtained without significant increase in the run-time of the standard HS algorithm. The other algorithm, called adaptive $H S$, refines the normal obtained by the integrated HS algorithm, by iteratively integrating the intensities over areas corresponding to the projection of the same surface point neighbourhood.

The experiments on two objects with non-trivial surface geometry and strong texture showed a significant increase in the quality of both the depth map and the normal field reconstructed, compared with the standard HS algorithm. It also resulted in a significant improvement in the consistency of the radiometric constraints used to validate the hypotheses on surface geometry. One limitation in our current implementation is that, the size of the convolution kernel used for filtering in the integrated HS algorithm, or the radius of the patch projected for the adaptive HS algorithm, have to be selected empirically. In future work we propose to investigate methods for adjusting automatically these sizes to the size of the structure or texture. The selection of the optimum size could for example be driven by the saliency; an appropriate size is expected to minimize the saliency values.

\section{Acknowledgments}

J.-Y. Guillemaut and J. Illingworth acknowledge the support by EPSRC project No GR/R08629/01. O. Drbohlav is supported by the Marie Curie Intra-European Fellowship No 506053 (Pho$\mathrm{Cal}$ ) within the 6th European Community Framework Programme. R. Sara acknowledges support by the Czech Ministry of Education under project MSM 212300013. This work has been initiated when O. Drbohlav was with the Center for Machine Perception, Prague, and J.-Y. Guillemaut was at his stay there under support of the Socrates exchange programme. The authors wish to thank the reviewers as their comments helped to improve the readability of this paper and the authors do profit from the insight provided. 

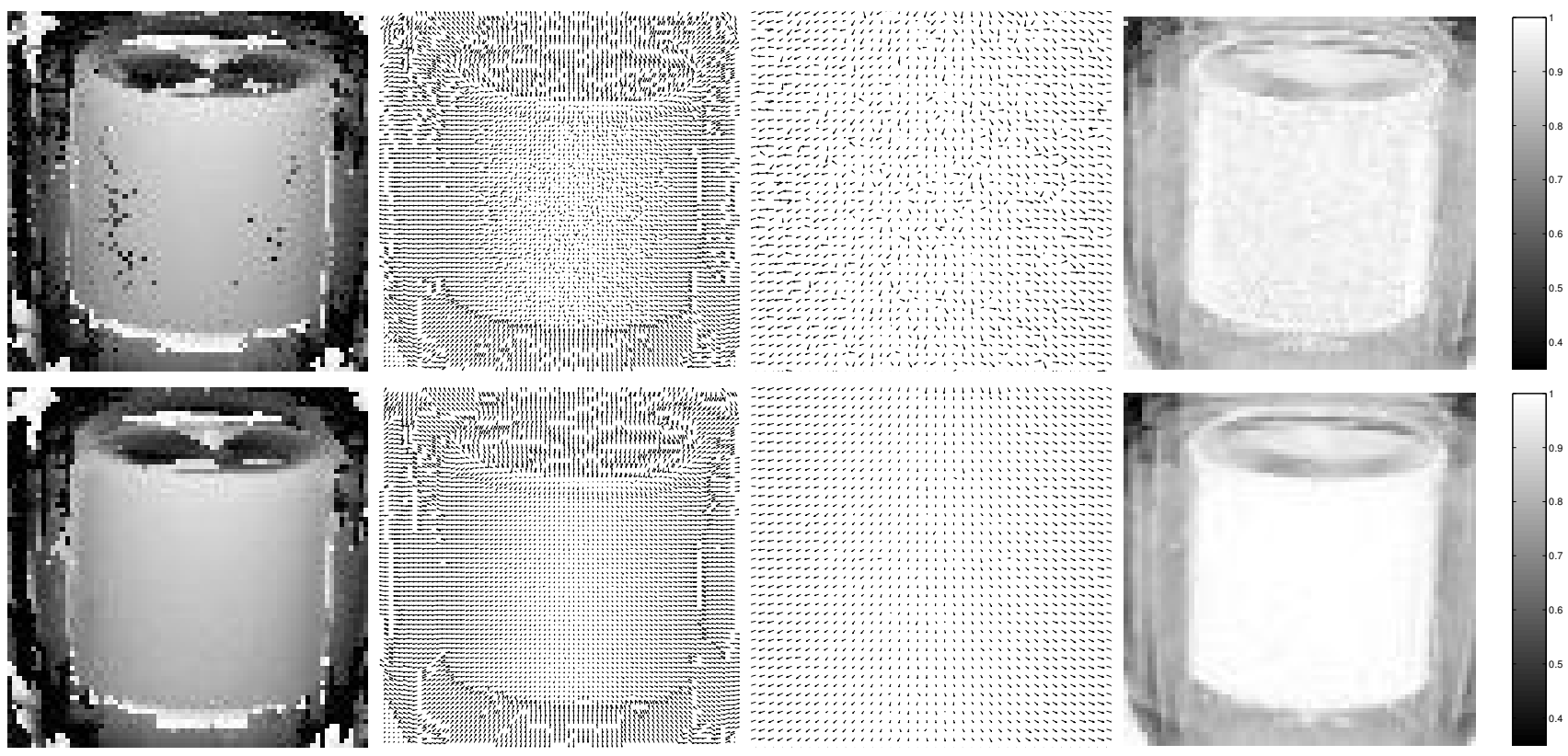

Figure 10: Reconstruction of the textured mug using the standard (top row) and the integrated (bottom row) HS algorithms: depth map (first column), normal field (second column), detail of the normal field for the face (third column), and saliency map (fourth column). The depth map and normals are again less noisy when consistent measurements of radiance are used. The saliency is also higher.

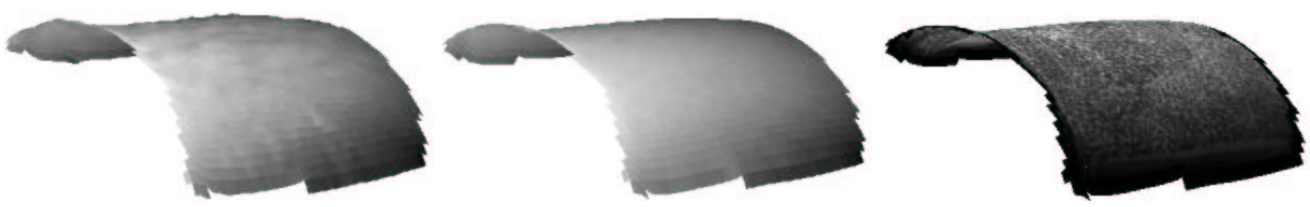

Figure 11: The surface of the textured mug reconstructed with the standard HS algorithm (left) and integrated HS algorithm (centre). The surfaces are obtained by integrating the normal fields shown in Fig. 10. The image on the right shows the surface reconstructed with the integrated HS algorithm and texture mapped with the left image from Fig. 9.

\section{References}

[1] Z. Janko, O. Drbohlav, and R. Sara. Radiometric calibration of a helmholtz stereo rig. In $C V P R, 2004$. To appear.

[2] J. H. Lambert. Photometria sive de mensura et gradibus luminis, colorum et umbra. Augustae Vindelicorum, Basel, 1760.

[3] S. Magda, D. J. Kriegman, T. Zickler, and P. N. Belhumeur. Beyond Lambert: Reconstructing surfaces with arbitrary BRDFs. In ICCV, volume 2, pages 391-398, 2001.

[4] F.E. Nicodemus, J.C. Richmond, J.J. Hsia, I.W. Ginsberg, and T. Limperis. Geometrical consideration and nomenclature for reflectance, 1977. NBS Monograph 160.

[5] W. C. Snyder. Definition and invariance properties of structured surface brdf. IEEE Transactions on Geoscience and Remote Sensing, 40(5):1032-1037, 2002.
[6] D. Terzopoulos. Multi-level reconstruction of visual surfaces: Variational principles and finite element representations. A.I. Memo 671, MIT, 1982.

[7] P. Tu and P. R. S. Mendonça. Surface reconstruction via Helmholtz reciprocity with a single image pair. In $C V P R$, volume 1, pages 541-547, 2003.

[8] T. E. Zickler, P. N. Belhumeur, and D. J. Kriegman. Helmholtz stereopsis: Exploiting reciprocity for surface reconstruction. IJCV, 49(2/3):215-227, 2002.

[9] T. E. Zickler, P. N. Belhumeur, and D. J. Kriegman. Toward a stratification of Helmholtz stereopsis. In CVPR, volume 1, pages 548-555, 2003.

[10] T. E. Zickler, J. Ho, D. J. Kriegman, J. Ponce, and P. N. Belhumeur. Binocular Helmholtz stereopsis. In $I C C V$, pages 1411-1417, 2003. 